\title{
Link invariants from finite biracks
}

\author{
Sam Nelson
}

\begin{abstract}
A birack is an algebraic structure with axioms encoding the blackboard-framed Reidemeister moves, incorporating quandles, racks, strong biquandles and semiquandles as special cases. In this paper we extend the counting invariant for finite racks to the case of finite biracks. We introduce a family of biracks generalizing Alexander quandles, $(t, s)$-racks, Alexander biquandles and Silver-Williams switches, known as $(\tau, \sigma, \rho)$-biracks. We consider enhancements of the counting invariant using writhe vectors, image subbiracks, and birack polynomials.
\end{abstract}

KEYworDs: Biracks, biquandles, Yang-Baxter equation, virtual knot invariants, enhancements of counting invariants

2000 MSC: 57M27, 57M25

\section{Introduction}

The modern study of algebraic structures with axioms corresponding to Reidemeister moves on knot diagrams goes back at least to the early 1980s with the more or less simultaneous work of Joyce [14 and Matveev 17, drawing in some cases on previous work by Takasaki [25] and Conway and Wraith [8].

Assigning algebraic generators to the arcs in a knot diagram and interpreting crossings as operations paves the way for translating Reidemeister moves into algebraic axioms. Applying this formula to unoriented link diagrams yields the involutory quandle or kei structure; generalizing to oriented link diagrams gives us the quandle or distributive groupoid structure. Generalizing again to blackboard-framed diagrams as in [10] gives us the rack or automorphic set structure.

A further generalization replaces arcs, i.e., portions of the knot diagram from one undercrossing point to another, with semiarcs, i.e., portions of the knot diagram from one over- or under-crossing point to the next. Semiarcs are edges in the graph obtained from a link diagram by replacing crossing points with vertices. Previous work has been done on the semiarc-generated algebraic structures arising from unframed oriented link diagrams, known as biquandles [2, 9, 15, 24. A special case of biquandles, applicable to flat virtual links or virtual strings and known as semiquandles, is examined in [13] with further examples appearing in [12].

In this paper we extend the method introduced in 23. of obtaining invariants of unframed classical and virtual knots and links from finite racks to the case of biracks, the algebraic structure generated by semiarcs in a link diagram with axioms corresponding to blackboard-framed isotopy, first introduced in [11. The new family of invariants contains the quandle, rack and strong biquandle counting invariants as special cases. In particular, the fundamental birack determines the knot quandle and fundamental rack and hence is complete invariant up to ambient homeomorphism for oriented framed knots and unsplit links; see 10, 14, 17.

The paper is organized as follows. In section 2 we define biracks and give a few examples. In section 3 we describe a family of biracks containing Alexander quandles, Alexander biquandles, $(t, s)$-racks and Silver-Williams switches as special cases. In section 4 we define a counting invariant of unframed classical knots and links using finite biracks and define enhancements of the birack counting invariants using image subbiracks, framing vectors and birack polynomials. We end with some questions for future research.

\section{Birack basics}

Let $X$ be a set. 
Definition 1 We will say a map $B: X \times X \rightarrow X \times X$ is strongly invertible provided $B$ satisfies the following three conditions:

- $B$ is invertible, i.e there exists a map $B^{-1}: X \times X \rightarrow X \times X$ satisfying $B \circ B^{-1}=\operatorname{Id}_{X \times X}=B^{-1} \circ B$,

- $B$ is sideways invertible, i.e there exists a unique invertible map $S: X \times X \rightarrow X \times X$ satisfying

$$
S\left(B_{1}(x, y), x\right)=\left(B_{2}(x, y), y\right),
$$

for all $x, y \in X$, and

- The sideways maps $S$ and $S^{-1}$ are diagonally bijective, i.e. the compositions $S_{1}^{ \pm 1} \circ \Delta, S_{2}^{ \pm 1} \circ \Delta$ of the components of $S$ and $S^{-1}$ with the map $\Delta: X \rightarrow X \times X$ defined by $\Delta(x)=(x, x)$ are bijections.

Definition 2 A birack $(X, B)$ is a set $X$ with a strongly invertible map $B: X \times X \rightarrow X \times X$ which satisfies the set-theoretic Yang-Baxter equation

$$
(B \times \mathrm{Id}) \circ(\mathrm{Id} \times B) \circ(B \times \operatorname{Id})=(\mathrm{Id} \times B) \circ(B \times \operatorname{Id}) \circ(\mathrm{Id} \times B)
$$

In previous work such as $[6,15,24$, the components of $B$ were interpreted as binary operations or rightactions of the set $X$ on itself, with $B_{1}(x, y)$ denoted by $y^{x}, B_{2}(x, y)=x_{y}, B_{1}^{-1}(x, y)=y_{\bar{x}}$ and $B_{2}^{-1}(x, y)=x^{\bar{y}}$. We can write the Yang-Baxter requirement as three equations in the component maps:

$$
\begin{aligned}
B_{1}\left(x, B_{1}(y, z)\right) & =B_{1}\left(B_{1}(x, y), B_{1}\left(B_{2}(x, y), z\right)\right) \\
B_{1}\left(B_{2}\left(x, B_{1}(y, z)\right), B_{2}(y, z)\right) & =B_{2}\left(B_{1}(x, y), B_{1}\left(B_{2}(x, y), z\right)\right) \\
B_{2}\left(B_{2}\left(x, B_{1}(y, z)\right), B_{2}(y, z)\right) & =B_{2}\left(B_{2}(x, y), z\right)
\end{aligned}
$$

or in the notation of [15],

$$
\left(z^{y}\right)^{x}=\left(z^{x_{y}}\right)^{y^{x}}, \quad\left(y_{z}\right)^{x_{z} y}=\left(y^{x}\right)_{z^{x_{y}}}, \quad \text { and } \quad\left(x_{z^{y}}\right)_{y_{z}}=\left(x_{y}\right)_{z} .
$$

Recall that a blackboard-framed link is an equivalence class of link diagrams under the equivalence relation generated by the three blackboard-framed Reidemeister moves, traditionally numbered according to the number of strands involved in the move:

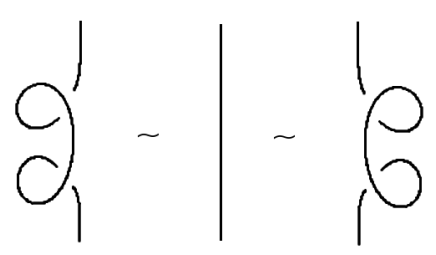

type I

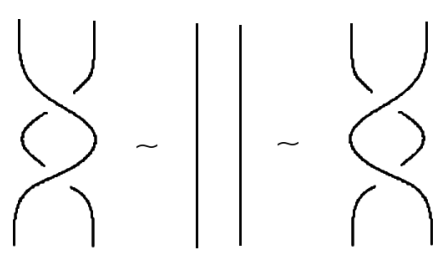

type II

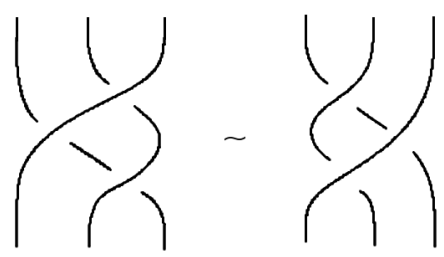

type III

The birack axioms are chosen such that any labeling of the semiarcs in an oriented blackboard-framed link diagram with elements of $X$ satisfying the identifications
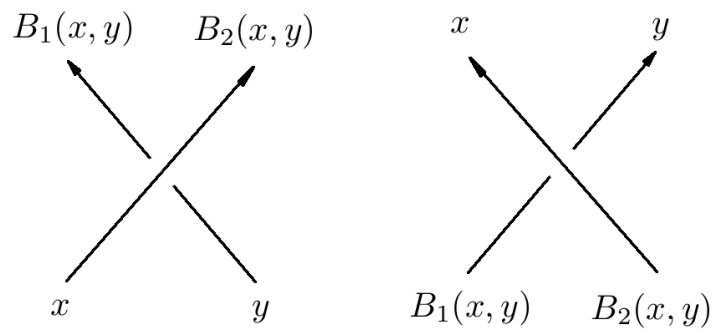
corresponds to a unique such labeling after applying any of the blackboard-framed Reidemeister moves. Thus, the number of such labelings is an easily computable invariant of blackboard-framed isotopy.

The condition that $B$ is a solution to the set-theoretic Yang-Baxter equation is equivalent to the condition that labelings are preserved by the type III move with Cartesian product $\times$ indicating horizontal stacking and composition o indicating vertical stacking.

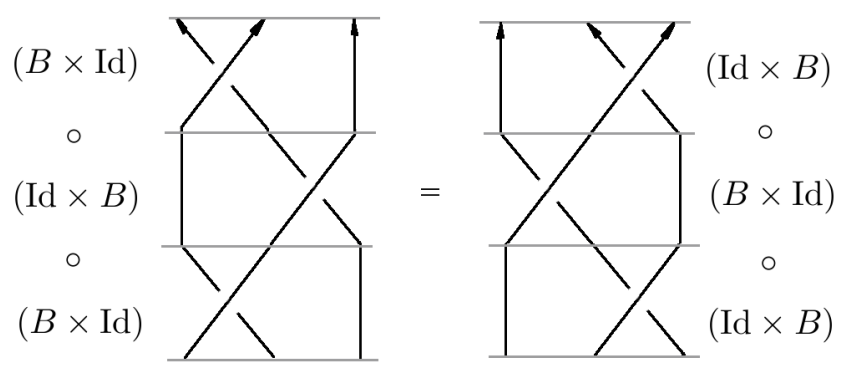

Interpreting $B^{-1}$ as the map defined at a negative crossing satisfies the direct Reidemeister II moves, i.e. the two two-strand moves where both strands are oriented in the same direction.
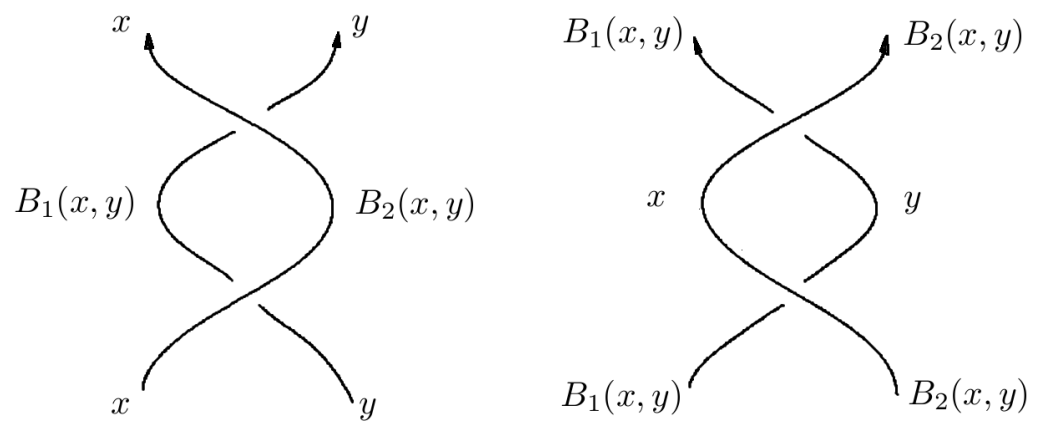

The sideways invertibility requirement is needed to guarantee the existence and uniqueness of the labels for the middle semiarcs in the reverse type II moves, where the strands are oriented in opposite directions:
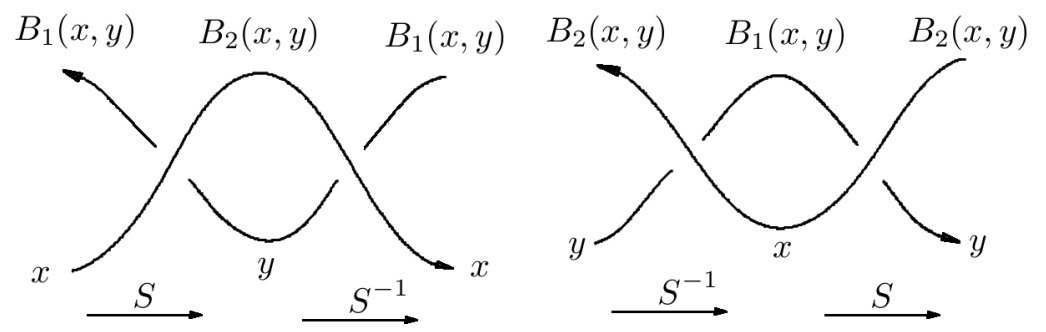

Remark 1 The existence, uniqueness and invertibility of the sideways map $S$ are equivalent to the requirement that the component maps $B_{1}$ and $B_{2}$ of $B$ are left- and right-invertible respectively; this condition is sometimes called the strong birack condition. Not imposing this requirement allows single labelings before a crossing-introducing reverse type II to branch into multiple labelings after the move, a situation we must avoid if we want well-defined counting invariants.

Remark 2 While there are eight total oriented Reidemeister III moves, the other seven moves follow from the pictured III move and direct and reverse II moves. Thus, the various identities required by the other type III moves are satisfied by a birack. 
The diagonal invertibility condition is required in order to guarantee the existence and uniqueness of the labels in the blackboard-framed type I moves. Of particular importance are the bijections $\alpha=\left(S_{2}^{-1} \circ \Delta\right)^{-1}$ and $\pi=S_{1}^{-1} \circ \Delta \circ \alpha$; these give the labels on a strand after a framed type I move as pictured.

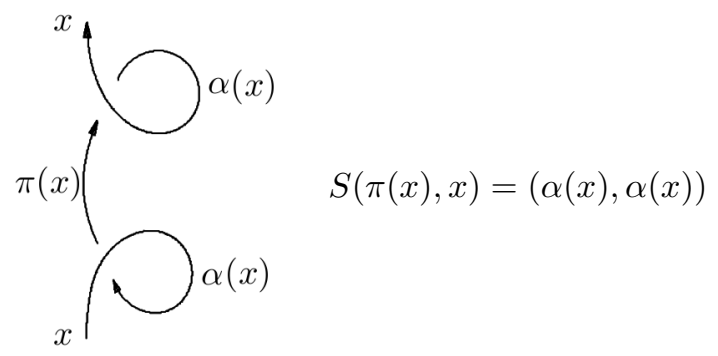

In fact, a priori we have two potentially distinct bijections $\pi$ and $\phi$ coming from the two oriented double-I moves. It turns out that the birack axioms imply that the two kink maps coincide:

Proposition 1 Let $(X, B)$ be a birack and $\phi, \pi: X \rightarrow X$ be the maps $\pi=S_{1}^{-1} \circ \Delta \circ\left(S_{2}^{-1} \circ \Delta\right)^{-1}$ and $\phi=S_{1} \circ \Delta \circ\left(S_{2} \circ \Delta\right)^{-1}$ as pictured. Then $\phi=\pi$.

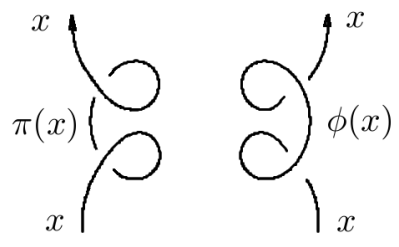

Proof. Since $(X, B)$ is birack, $B$ satisfies the set-theoretic Yang-Baxter equation and $S$ satisfies the invertibility requirements for type II moves. Thus, labelings of knot diagrams by elements of $X$ satisfying the birack labeling condition are preserved under regular isotopy. Then the fact that (see [10] for instance) oppositewrithe opposite-winding-number twists can be canceled using only type II and type III moves implies that $\phi^{-1}(\pi(x))=x$ for all $x \in X$, and we have $\phi=\pi$.

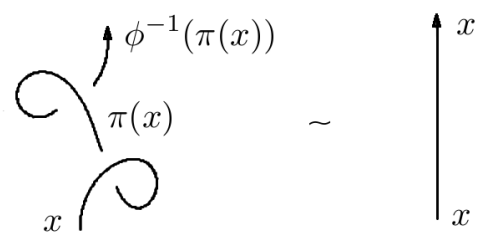

Definition 3 Let $(\mathrm{X}, \mathrm{B})$ be a birack. Then the bijection $\pi: X \rightarrow X$ given by $\pi=S_{1}^{-1} \circ \Delta \circ\left(S_{2}^{-1} \circ \Delta\right)^{-1}$ is the kink map of $(X, B) . \pi(x)$ represents "going through a positive kink" while $\pi^{-1}$ represents "going through a negative kink".

Next we have a few basic definitions relating to the algebra of biracks.

Definition 4 Let $Y$ be a subset of $X$. Then $Y$ is a subbirack of $(X, B)$ if the images of the restrictions of the components of $B$ and $S$ to $Y \times Y$ are contained in $Y$. 
Definition 5 Let $(X, B)$ and $\left(X^{\prime}, B^{\prime}\right)$ be biracks. Then a map $h: X \rightarrow X^{\prime}$ is a birack homomorphism if for all $x, y \in X$ we have

$$
h\left(B_{1}(x, y)\right)=B_{1}^{\prime}(h(x), h(y)) \text { and } \quad h\left(B_{2}(x, y)\right)=B_{2}^{\prime}(h(x), h(y)) .
$$

For any birack homomorphism $h: X \rightarrow X^{\prime}$ the image of $h$, denoted $\operatorname{Im}(h)$, is the subbirack of $X^{\prime}$ generated by the elements $h(x)$ for $x \in X$.

Definition 6 Let $(X, B)$ be a birack and let $\pi: X \rightarrow X$ be the kink map. The birack rank of $(X, B)$, denoted $N(X, B)$ or just $N$ when $(X, B)$ is clear from context, is the smallest positive integer $N$ such that $\pi^{N}(x)=x$ for all $x \in X$. In particular, if $X$ is a finite set, then $N$ is the exponent of $\pi$ considered as an element of the symmetric group $S_{X}$.

If $N(X, B)<\infty$ then there is a bijection between the sets of labelings of any two oriented blackboardframed link diagrams $D$ and $D^{\prime}$ which are related by blackboard framed moves together with the $N$-phone cord move:

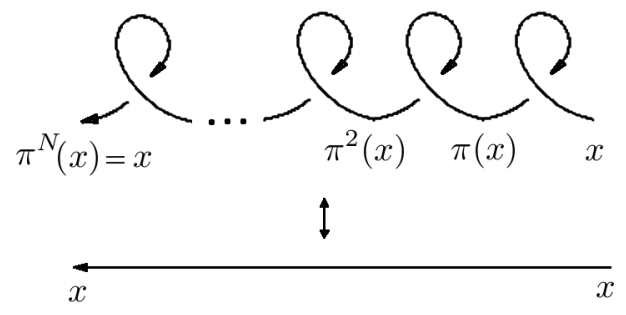

Example 1 Let $X$ be any set, and let $\rho, \tau: X \rightarrow X$ be bijections. Then $B(x, y)=(\tau(y), \rho(x))$ defines a birack provided $\rho$ and $\tau$ commute. The reader is invited to verify that the Yang-Baxter equation components become

$$
\rho^{2}(x)=\rho^{2}(x), \quad \tau \rho(y)=\rho \tau(y), \quad \text { and } \quad \tau^{2}(z)=\tau^{2}(z) .
$$

Moreover, we have $B^{-1}(x, y)=\left(\rho^{-1}(y), \tau^{-1}(x)\right), S(u, v)=\left(\rho(v), \tau^{-1}(u)\right)$ with $S^{-1}(u, v)=\left(\tau(v), \rho^{-1}(u)\right)$, $f(x)=\tau^{-1}(x)$ and $g(x)=\rho(x)$. We call a birack of this type a constant action birack. The kink map is $\pi(x)=\rho \tau^{-1}(x)$, so $N(X, B)$ is the order of the permutation $\rho \tau^{-1}$ in $S_{|X|}$. In particular, a constant action birack is a strong biquandle iff $\rho=\tau$.

Example 2 Any oriented blackboard-framed link diagram $L=L_{1} \cup \cdots \cup L_{c}$ has a fundamental birack denoted $B R(L)$ (or $B R\left(L,\left(w_{1}, \ldots, w_{c}\right)\right)$ if we wish to explicitly specify the writhe numbers of the components). Let $G=\left\{g_{1}, \ldots, g_{n}\right\}$ correspond to the semiarcs of $L$. Define the set $B W(G)$ of birack words recursively by $x \in B W(G)$ iff $x \in G, x=B_{i}^{ \pm 1}(y, z), x=S_{i}^{ \pm 1}(y, z), x=f^{ \pm 1}(y)$ or $x=g^{ \pm 1}(y)$ where $y, z \in B W(G)$ and $i \in\{1,2\}$. Then the free birack on $G$ is the set of equivalence classes of $B W(G)$ under the equivalence relation generated by the various equations coming from the Yang-Baxter equation and the strong invertibility requirements (e.g., $g(x) \sim S_{1}(x, x)$, etc.). The fundamental birack $B R(L)$ of the link $L$ is then the set of equivalence classes of free blackboard birack elements under the additional equivalence relation generated by the crossing relations in $L$. We will generally express the birack of $G$ by $B R(L)=\langle G \mid R\rangle$ where $G$ is the set of arc labels and $R$ is the set of crossing relations, e.g.

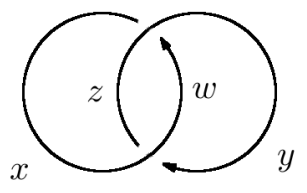

$$
B R(L)=\langle x, y, z, w \mid B(x, y)=(z, w), B(z, w)=(x, y)\rangle .
$$


The quotient of $B R(L)$ obtained by setting $B_{2}(x, y)$ equal to $x$ in all relations is the fundamental rack $F R(L)$ of the blackboard-framed link $L$; the quotient of $B R(L)$ obtained by setting $\pi(x)=x$ for all $x \in B R(L)$ is the fundamental (strong) biquandle of $L$. Imposing both conditions yields the knot quandle. In particular, since $B R(L)$ determines the fundamental rack of $L$, the isomorphism class of $B R(L)$ is a complete invariant in the same sense as the fundamental rack and the knot quandle; see [10, 14, 17.

Remark 3 The cardinality of the set of labelings of a blackboard-framed link diagram $L$ by a birack $(X, B)$ satisfying the labeling condition above is an invariant of blackboard framed isotopy we call the basic counting invariant, denoted

$$
|\operatorname{Hom}(B R(L),(X, B))|=\mid\{h \mid h: B R(L) \rightarrow X \text { birack homomorphism }\} \mid .
$$

Indeed, each labeling satisfying the crossing condition determines a unique homomorphism $f: B R(L) \rightarrow X$ and every birack homomorphism corresponds to a unique labeling of $L$.

Example 3 Many previously studied algebraic structures are special cases of biracks.

- A birack with $\pi=\mathrm{Id}: X \rightarrow X$ is a (strong) biquandle (see [15, 24, 9]);

- A birack with $\pi=\mathrm{Id}$ and $B^{-1}=B$ is a semiquandle (see [13]);

- A birack with $B_{2}(x, y)=x$ is a rack (see [10, 23]);

- A birack with both $B_{2}(x, y)=x$ and $\pi=\mathrm{Id}$ is a quandle (see [14]).

To define birack structures on a finite set $X=\left\{x_{1}, \ldots, x_{n}\right\}$ without algebraic formulas for the component maps $B_{i}$, we can give a pair of $n \times n$ matrices with entries in $\{1,2 \ldots, n\}$ encoding the component maps of $B$. Specifically, the birack matrix of a birack $X$ is the block matrix $M_{B}=\left[B_{1} \mid B_{2}\right]$ such that the entries in row $i$ column $j$ of $B_{1}$ and $B_{2}$ respectively are $x_{k}$ and $x_{h}$ where $B_{1}\left(x_{i}, x_{j}\right)=x_{k}$ and $B_{2}\left(x_{j}, x_{i}\right)=x_{h}$. Note the transposition of the input variables for $B_{2}$; this is for backwards compatibility with notation from previous work. Comparing the notation from [24, our $B_{1}$ is the upper right block matrix and our $B_{2}$ is the lower right block matrix.

Example 4 As an easy example, let $X=\{1,2,3,4\}$ and let $\tau=(12)$ and $\rho=(34)$. Then $\rho \tau=\tau \rho$ and we have a birack with matrix

$$
M_{B}=\left[\begin{array}{llll|llll}
2 & 2 & 2 & 2 & 1 & 1 & 1 & 1 \\
1 & 1 & 1 & 1 & 2 & 2 & 2 & 2 \\
3 & 3 & 3 & 3 & 4 & 4 & 4 & 4 \\
4 & 4 & 4 & 4 & 3 & 3 & 3 & 3
\end{array}\right] .
$$

The kink map here is $\pi=(12)(34)$, and thus we have birack rank $N=2$.

\section{$3 \quad(t, s, r)$-biracks and $(\tau, \sigma, \rho)$-biracks}

In this section we define two classes of biracks, $(t, s, r)$-biracks and $(\tau, \sigma, \rho)$-biracks. We begin with the simpler case. Let $\tilde{\Lambda}=\mathbb{Z}\left[t^{ \pm 1}, s, r^{ \pm 1}\right] / I$ where $I$ is the ideal generated by $s^{2}-(1-t r) s$. Let $X$ be any $\tilde{\Lambda}$ module.

Lemma 2 The element $\pi=t r+s \in \tilde{\Lambda}$ is invertible with inverse given by $\pi^{-1}=t^{-1} r^{-1}(1-s)$.

Proof.

$$
\begin{aligned}
(t r+s) t^{-1} r^{-1}(1-s) & =(t r+s)\left(t^{-1} r^{-1}-t^{-1} r^{-1} s\right) \\
& =1+t^{-1} r^{-1} s-s-t^{-1} r^{-1} s^{2} \\
& =1+t^{-1} r^{-1} s-s-t^{-1} r^{-1}(1-t r) s \\
& =1+\left(t^{-1} r^{-1}-1\right) s-t^{-1} r^{-1}(1-t r) s \\
& =1+\left(t^{-1} r^{-1}-1\right) s-\left(t^{-1} r^{-1}-1\right) s \\
& =1 .
\end{aligned}
$$


Corollary 3 The element $(1-s) \in \tilde{\Lambda}$ is invertible with inverse $(1-s)^{-1}=1+t^{-1} r^{-1} s$.

Proposition 4 Let $X$ be a $\tilde{\Lambda}$-module and define $B(x, y)=(t y+s x, r x)$. Then $(X, B)$ is a birack with kink $\operatorname{map} \pi(x)=(t r+s) x$.

\section{Proof.}

We must check that $B$ is strongly invertible. First, note that $B^{-1}(x, y)=\left(r^{-1} x, t^{-1} y-s t^{-1} r^{-1} x\right)$ so $B$ is invertible. Next, the sideways map $S$ is given by $S(x, y)=\left(r y, t^{-1} x-t^{-1}\right.$ sy) with inverse $S^{-1}(x, y)=$ $\left(t y+s r^{-1} x, r^{-1} x\right)$. Finally, for diagonal invertibility, we have $f(x)=t^{-1}(1-s) x$ and $g(x)=r(x)$, so $\pi(x)=\left(g \circ f^{-1}\right)(x)=t(1-s)^{-1} r x=t r\left(1+t^{-1} r^{-1} s\right) x=(t r+s) x$.

Next, we must check that the set-theoretic Yang-Baxter equation is satisfied. In component form, we have

$$
\begin{aligned}
t^{2} z+t s y+s x & =t^{2} z+s t y+\left(s t r+s^{2}\right) x \\
r(t y+s x) & =t(r y)+s(r x) \\
r^{2} x & =r^{2} x .
\end{aligned}
$$

Commutativity in $\tilde{\Lambda}$ satisfies the second equation and reduces the first equation to $(1-t r) s=s^{2}$.

For the purpose of defining counting invariants with $(t, s, r)$-biracks, we'll need finite examples. The simplest way to do this is to choose a finite abelian group, say $A=\mathbb{Z}_{n}$, and choose elements $t, s, r \in A$ such that $t, r$ are invertible and $s^{2}=(1-t r) s$. Then the set $A^{m}$ of $m$-tuples of $A$ forms a finite $(t, s, r)$-rack.

Corollary 5 A finite $(t, s, r)$-birack structure $(A, B)$ on an abelian group $A$ is a strong biquandle iff tr $+s=1$.

Corollary 6 The birack rank of a finite $(t, s, r)$-birack is the smallest integer $N>0$ such that $(t r+s)^{N}=1$.

Example 5 Let $A=\mathbb{Z}_{4}$ and set $t=3, s=2$ and $r=3$. Then $s^{2}=4=0=2(1-9)=s(1-t r)$ so $A^{m}$ is a finite $(t, s, r)$-birack with birack operation $B(x, y)=(3 y+2 x, 3 x) .\left|A^{m}\right|=4^{m}$ and $A_{m}$ has birack rank 2 since $t r+s=9+2=3$ and $3^{2}=9=1$.

Remark $4(t, s, r)$-biracks have several important special cases. If we set $r=1$, we have a $(t, s)$-rack as defined in [10. introduced in [10] and subsequently studied in papers such as [7]. In the case $s=1-\operatorname{tr}$ we have a biquandle known as an Alexander biquandle, introduced in [15] and subsequently studied in papers such as [16. If we set $r=1$ and $s=1-t$, we have an Alexander quandle, introduced in [14] and studied in many papers such as $[1,18$.

Remark 5 The general case of biquandle structures defined via linear operations on modules over noncommutative rings has been studied in several recent papers [3, 4, 12. Certain cases of these (known as quaternionic biquandles) yield invariants of virtual knots analogous to the Alexander polynomial.

Every $\tilde{\Lambda}$-module is an abelian group under addition with multiplication by $t$ and $r$ acting as automorphisms of the additive structure and multiplication by $s$ acting as an endomorphism. This suggests a way to generalize the $(t, s, r)$-birack definition by unabelianizing the group structure.

Proposition 7 Let $G$ be a group with $\tau, \rho \in \operatorname{Aut}(G)$ and $\sigma \in \operatorname{End}(G)$ such that $\rho$ commutes with $\tau$ and $\sigma$ and satisfying for all $y, z \in G$

$$
\tau \sigma(y) \sigma(z)=\tau \sigma \rho(z) \sigma \tau(y) \sigma^{2}(z) .
$$

Define $B: G \times G \rightarrow G \times G$ by $B(x, y)=(\tau(y) \sigma(x), \rho(x))$. Then $(G, B)$ is a birack with kink map given by $\pi(x)=\tau \rho(x) \sigma(x)$; we call this a $(\tau, \sigma, \rho)$-birack. 
Proof. It is a straightforward computation to show that

$$
\begin{aligned}
B^{-1}(x, y) & =\left(\rho^{-1}(y), \tau^{-1}\left(x\left(\sigma \rho^{-1}(y)\right)^{-1}\right)\right), \\
S(x, y) & =\left(\rho(y), \tau^{-1}\left(x \sigma(y)^{-1}\right)\right) \\
S^{-1}(x, y) & =\left(\tau(y) \sigma\left(\rho^{-1}(x)\right), \rho^{-1}(y)\right)
\end{aligned}
$$

and that we have diagonal invertibility with kink map given by $\pi(x)=\tau \rho(x) \sigma(x)$.

To see that the set-theoretic Yang-Baxter equation is satisfied, we note that $B$ gives us the Yang-Baxter component form equations

$$
\begin{aligned}
\tau^{2}(x) \tau \sigma(y) \sigma(z) & =\tau^{2}(x) \tau \sigma \rho(z) \sigma \tau(y) \sigma^{2}(z) \\
\tau \rho(y) \sigma \rho(z) & =\rho \tau(y) \rho \sigma(z) \\
\rho^{2}(z) & =\rho^{2}(z) .
\end{aligned}
$$

The first equation reduces to equation (4) and the second requires that $\rho$ commute with $\sigma$ and $\tau$.

A few special cases of $(\tau, \sigma, \rho)$-biracks appear in the literature; the case where the kink map $\pi(x)=\operatorname{Id}(x)$ is a biquandle known as a Silver-Williams switch [9, and in the quandle case, i.e. $\rho(x)=\operatorname{Id}(x)$ and $\sigma(x)=\tau\left(x^{-1}\right) x$, we have a quandle structure incorrectly referred to by the present author in previous work as "homogeneous quandles"; a better term would be multiplicative Alexander quandles as these need not be homogeneous in the sense of [14]. The special case where $G$ is the automorphism group $\operatorname{Aut}(Q)$ of a quandle $Q$ and $\tau$ is conjugation in $G$ by a chosen inner automorphism turns out to be the key to the relationship between the knot group and the knot quandle; see [14, 17] for more.

Example 6 Let $G=\left\langle\alpha, \beta \mid \alpha^{4}=1, \beta^{m}=1, \alpha \beta=\beta \alpha^{-1}\right\rangle$ and let $\tau\left(\alpha^{i} \beta^{j}\right)=\rho\left(\alpha^{i} \beta^{j}\right)=\beta^{j} \alpha^{i}$ and $\sigma\left(\alpha^{i} \beta^{j}\right)=\alpha^{2 i}$. Then $\tau, \rho \in \operatorname{Aut}(G), \sigma \in \operatorname{End}(G)$ and we have $\rho \tau=\operatorname{Id}=\tau \rho \quad$ and $\quad \rho \sigma=\sigma=\sigma \rho$. Then for $y=\alpha^{i} \beta^{j}$ and $z=\alpha^{k} \beta^{l}$, we have

$$
\tau \sigma(y) \sigma(z)=\alpha^{2 i} \alpha^{2 k}=\alpha^{2 k} \alpha^{2 i} \alpha^{4 k}=\tau \sigma \rho(z) \sigma \tau(y) \sigma^{2}(z)
$$

and thus we have a $(\tau, \sigma, \rho)$-birack. Here the kink map is given by $\pi\left(\alpha^{i} \beta^{j}\right)=\alpha^{3 i} \beta^{j}$, so we have birack rank $N=2$.

\section{Birack link invariants}

By construction, there is a bijection between the sets of labelings of any two blackboard-framing-isotopic link diagrams by the same birack $(X, B)$. In particular, as we increment the writhe of the components in the diagram, the number of labelings of a knot diagram by a birack has period $N$ in each component where $N$ is the birack rank. As in [23, we can sum these basic counting invariants over a complete period of framings to define an invariant of unframed ambient isotopy classes of oriented links, provided the labeling birack $X$ is finite. In particular, a link $L=L_{1} \cup \cdots \cup L_{c}$ of $c$ components has writhe vectors in $W=\left(\mathbb{Z}_{N}\right)^{c}$ with respect to the labelings by a blackboard birack $(X, B)$.

Definition 7 Let $(X, B)$ be a birack with birack rank $N, L=L_{1} \cup \cdots \cup L_{c}$ a $c$-component link and $W=\left(\mathbb{Z}_{N}\right)^{c}$. Then the integral birack counting invariant of $L$ with respect to $(X, B)$ is

$$
\phi_{(X, B)}^{\mathbb{Z}}(L ;(X, B))=\sum_{\mathbf{w} \in W}|\operatorname{Hom}(B R(L, \mathbf{w}), X)| .
$$

Remark 6 If we define blackboard-framed isotopy of virtual links in the obvious way, namely as the result of replacing the classical type I moves with blackboard-framed double type I moves and keeping all other moves the same, then ignoring virtual crossings lets us extend the counting invariant and its enhancements below to virtual knots without modification. 
Example 7 The smallest birack which is neither a biquandle nor a rack is the two-element constant action birack $X=\{1,2\}$ with $\tau=\operatorname{Id}_{X}$ and $\rho=(12)$, i.e., with birack matrix

$$
M_{B}=\left[\begin{array}{ll|ll}
1 & 1 & 2 & 2 \\
2 & 2 & 1 & 1
\end{array}\right] .
$$

Interpreted as labeling rules, $(X, B)$ says we switch from 1 to 2 or 2 to 1 when going over a crossing and keep the same label when going under a crossing. In particular, the kink map $\pi$ is the transposition (12) so we have birack rank $N=2$. The counting invariant $\phi_{B R}^{\mathbb{Z}}(L)$ of a link $L$ is then the total number of colorings over a complete set of diagrams of $L$ with every combination of even and odd writhes on each component. Thus, both the Hopf link and the unlink of two components have four labelings by $(X, B)$

\begin{tabular}{|c|c|c|c|}
\hline $\mathbf{w}=(0,0)$ & $\mathbf{w}=(1,0)$ & $\mathbf{w}=(0,1)$ \\
\hline \\
\hline$x$
\end{tabular}

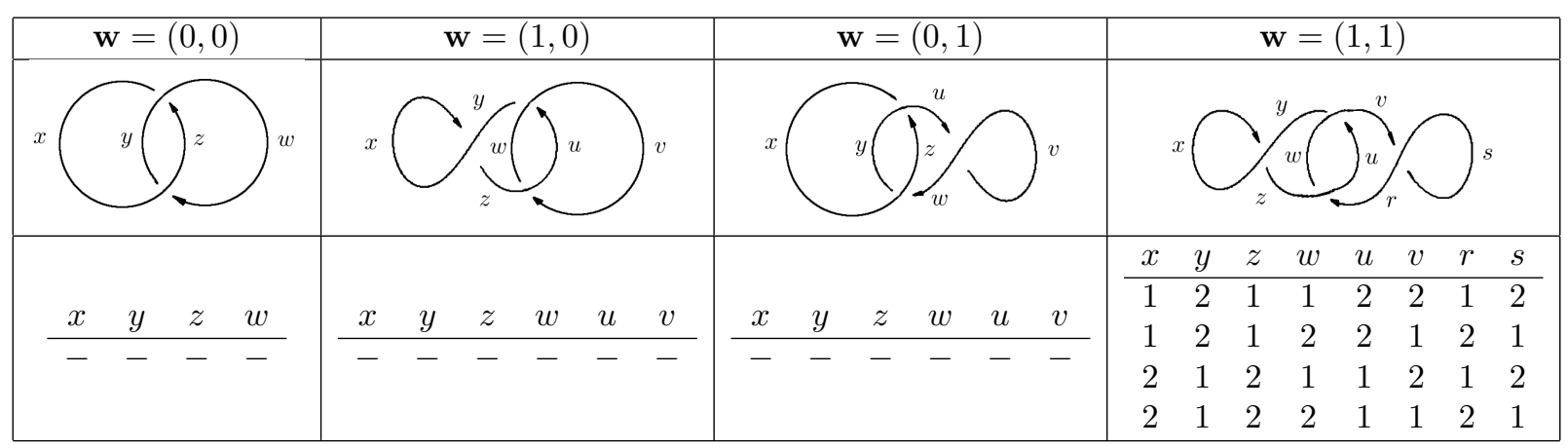

We notice that the total numbers of labelings of both links over one framing period $\bmod N$ are the same, but the labelings occur in different framings. To exploit this information, we will need to enhance the counting invariant.

An enhancement of a counting invariant is an invariant which specializes to the counting invariant but includes extra information to distinguish between labelings, making enhanced invariants stronger than the original counting invariants. One way to enhance counting invariants is to define a "signature" for each labeling which is well-defined under Reidemeister moves on the labeled diagram; the resulting multiset of signatures is then an enhancement of the counting invariant. Examples include using quandle 2-cocycles to define a Boltzmann weight signature for quandle labelings of $L$ (see [5]) as well as using extra structure of the labeling object to define signatures (see [19, 22] etc.). Taking a generating function lets us express these multiset-valued invariants as polynomials by converting multiplicities to coefficients and signatures to exponents, e.g. the multiset $\{0,0,1,1,1,2\}$ becomes the polynomial $2+3 z+z^{2}$.

As with the rack counting invariant, one easy type of enhancement involves keeping track of which framings contribute which labelings. Let $q^{\mathbf{w}}=\prod_{i=1}^{c} q_{i}^{w_{i}}$ for $\mathbf{w}=\left(w_{1}, \ldots, w_{c}\right) \in W$. Then we have:

Definition 8 Let $(X, B)$ be a birack with birack rank $N, L=L_{1} \cup \cdots \cup L_{c}$ a $c$-component link and $W=\left(\mathbb{Z}_{N}\right)^{c}$. The writhe-enhanced birack counting invariant of $L$ with respect to $(X, B)$ is

$$
\phi_{(X, B)}^{W, M}(L)=\{(|\operatorname{Hom}(B R(L, \mathbf{w}),(X, B))|, \mathbf{w}): \mathbf{w} \in W\}
$$


in multiset form; in polynomial form we have:

$$
\phi_{(X, B)}^{W}(L)=\sum_{\mathbf{w} \in W}|\operatorname{Hom}(B R(L, \mathbf{w}), X)| q^{\mathbf{w}} .
$$

Example 8 The Hopf link and two-component unlink, while not distinguished by the integral counting invariant determined by the birack in example 7, are distinguished by the writhe-enhanced invariant determined by the same birack, with $\phi_{(X, B)}^{W}($ Hopf $)=4 q_{1} q_{2} \neq 4=\phi_{(X, B)}^{W}($ Unlink $)$.

Remark 7 In the case of birack rank $N=1$, i.e. if $(X, B)$ is a quandle or strong biquandle, the integral and writhe-enhanced polynomial invariants are the same; indeed, in this case both consist of single basic counting invariant $|\operatorname{Hom}(B R(L),(X, B))|$. If $B_{2}(x, y)=x$ so that $(X, B)$ is a rack, then the integral and writheenhanced polynomial birack counting invariants are the integral and polynomial rack counting invariants described in $[23$.

Another straightforward enhancement uses the cardinality of the image subbirack as a signature:

Definition 9 Let $(X, B)$ be a birack with birack rank $N, L=L_{1} \cup \cdots \cup L_{c}$ a $c$-component link and $W=\left(\mathbb{Z}_{N}\right)^{c}$. The image-enhanced birack counting invariant of $L$ with respect to $(X, B)$ is

$$
\phi_{(X, B)}^{\operatorname{Im}, M}(L)=\{|\operatorname{Im}(f)|: f \in \operatorname{Hom}(B R(L, \mathbf{w}),(X, B)), \mathbf{w} \in W\}
$$

in multiset form; in polynomial form we have:

$$
\phi_{(X, B)}^{\operatorname{Im}}(L)=\sum_{\mathbf{w} \in W}\left(\sum_{f \in \operatorname{Hom}(B R(L, \mathbf{w}),(X, B))} z^{|\operatorname{Im}(f)|}\right) .
$$

Example 9 Let $L$ be the trefoil knot $3_{1}$ and $(X, B)$ the $(t, s, r)$-birack structure on $X=\mathbb{Z}_{3}$ given by $B(x, y)=(y+2 x, 2 x)$. Here the kink map is $\pi(x)=(t r+s) x=(2+2) x=x$ so we have $N=1$ and can compute the invariant from a diagram with any writhe. Since $\mathbb{Z}_{3}$ is a field, we can find the $\mathbb{Z}_{3}$-vector space of labelings of $L$ using linear algebra:

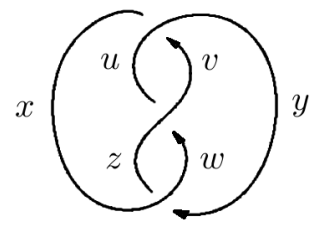

We have a presentation $B R\left(3_{1}\right)=\langle x, y, z, w, u, v \mid B(x, y)=(z, w), B(z, w)=(u, v), B(u, v)=(x, y)\rangle$. This translates into a coefficient matrix for a homogeneous system of linear equations, which we row-reduce over $\mathbb{Z}_{3}:$

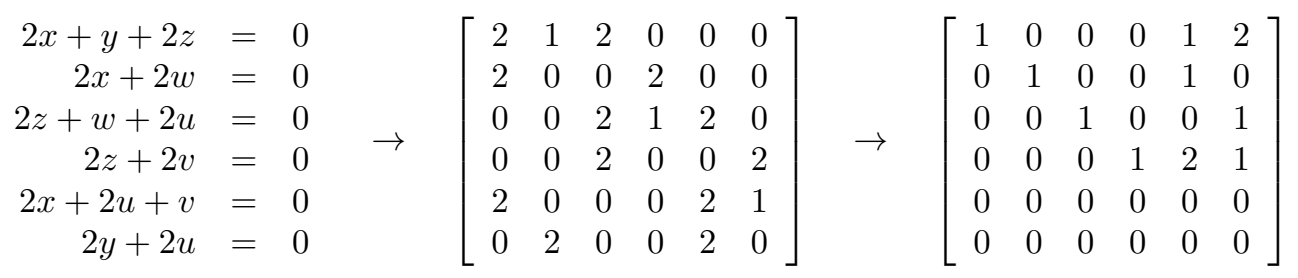

Thus we can see that the set of labelings is the $\mathbb{Z}_{3}$-span of the set $\{(1,1,0,2,2,0),(2,0,1,1,0,2)\}$; thus, eight of the $3^{2}=9$ labelings considered as birack homomorphisms $f: B R\left(3_{1}\right) \rightarrow\left(\mathbb{Z}_{3}, B\right)$ are surjective, while the trivial linear combination represents the zero homomorphism. Thus, the integral counting invariant is $\phi_{\left(\mathbb{Z}_{3}, B\right)}^{\mathbb{Z}}\left(3_{1}\right)=9$ while the image-enhanced invariant is $\phi_{\left(\mathbb{Z}_{3}, B\right)}^{\operatorname{Im}}\left(3_{1}\right)=1+8 z^{3}$. Similar computations yield $\phi_{\left(\mathbb{Z}_{3}, B\right)}^{\operatorname{Im}}($ Unknot $)=1+2 z^{3}$ and $\phi_{\left(\mathbb{Z}_{3}, B\right)}^{\operatorname{Im}}\left(4_{1}\right)=1$. 
Remark 8 As the above example illustrates, the set of labelings of a knot or link by a $(t, s, r)$-birack

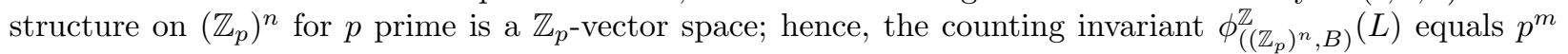
for some $m \geq 0$.

Remark 9 Say that a birack is simple if it has no non-empty proper subbiquandles, e.g. the birack in example 7. Then for simple biracks, the integral and image-enhanced invariants contain equal information; more precisely, for simple biracks we have

$$
\phi_{(X, B)}^{\mathbb{Z}}(L) z^{|X|}=\phi_{(X, B)}^{\operatorname{Im}}(L) .
$$

Note that if $(X, B)$ is a quandle then singletons are proper subbiracks, so quandles with $|X| \geq 2$ are not simple biracks.

For our final enhancement of the birack counting invariants, we will use birack polynomials. In [20] a two-variable polynomial invariant is defined for finite quandles and extended to biquandles in 21 and racks in $\underline{6}$. These polynomials quantify the way in which the trivial action of elements of $X$ on $X$ is distributed throughout the birack, as opposed to being concentrated in a single identity element as in a group. We will use a simplified version of the biquandle polynomial to define an enhancement of the birack counting invariant.

Definition 10 Let $(X, B)$ be a finite birack. For each $x \in X$ let

$$
\begin{gathered}
c_{1}(x)=\left|\left\{y \in X: B_{1}(y, x)=y\right\}\right|, \quad c_{2}(x)=\left|\left\{y \in X: B_{2}(x, y)=y\right\}\right|, \\
r_{1}(x)=\left|\left\{y \in X: B_{1}(y, x)=x\right\}\right|, \quad \text { and } \quad r_{2}(x)=\left|\left\{y \in X: B_{2}(x, y)=x\right\}\right| .
\end{gathered}
$$

The birack polynomial of $(X, B)$, denoted $\rho_{(X, B)}\left(s_{1}, t_{1}, s_{2}, t_{2}\right)$, is the four-variable polynomial

$$
\rho_{(X, B)}\left(s_{1}, s_{2}, t_{1}, t_{2}\right)=\sum_{x \in X} s_{1}^{c_{1}(x)} s_{2}^{c_{2}(x)} t_{1}^{r_{1}(x)} t_{2}^{r_{2}(x)} .
$$

Proposition 8 If $(X, B)$ and $\left(X^{\prime}, B^{\prime}\right)$ are isomorphic biracks, then

$$
\rho_{(X, B)}\left(s_{1}, s_{2}, t_{1}, t_{2}\right)=\rho_{\left(X^{\prime}, B^{\prime}\right)}\left(s_{1}, t_{1}, s_{2}, t_{2}\right) .
$$

Proof. If $\psi: X \rightarrow X^{\prime}$ is an isomorphism, then $c_{i}(\psi(x))=c_{i}(x)$ and $r_{i}(\psi(x))=r_{i}(x)$ for $i=1,2$.

Next, we have a polynomial for subbiracks $Y \subset X$. This polynomial contains information not just about the subbirack $Y$ itself but also about how $Y$ is embedded in $X$. See [20] for more.

Definition 11 For any subbirack $Y \subset X$ of $(X, B)$, the subbirack polynomial of $Y$ is given by

$$
\rho_{Y \subset X}\left(s_{1}, s_{2}, t_{1}, t_{2}\right)=\sum_{y \in Y} s_{1}^{c_{1}(y)} s_{2}^{c_{2}(y)} t_{1}^{r_{1}(y)} t_{2}^{r_{2}(y)} .
$$

Example 10 The birack with matrix

$$
M_{b}=\left[\begin{array}{llll|llll}
2 & 2 & 1 & 1 & 2 & 2 & 1 & 1 \\
1 & 1 & 2 & 2 & 1 & 1 & 2 & 2 \\
3 & 4 & 3 & 3 & 4 & 3 & 4 & 4 \\
4 & 3 & 4 & 4 & 3 & 4 & 3 & 3
\end{array}\right]
$$

has birack polynomial $\rho_{(X, B)}=s_{1}^{2} t_{1}^{2} t_{2}^{2}+s_{2}^{2} t_{1}^{2} t_{2}^{2}+2 s_{1}^{4} s_{2}^{2} t_{1}^{3} t_{2}$. There are two subbiracks, $Y=\{1,2\}$ and $Z=\{3,4\}$ with subbirack polynomials $\rho_{Y \subset X}=s_{1}^{2} t_{1}^{2} t_{2}^{2}+s_{2}^{2} t_{1}^{2} t_{2}^{2}$ and $\rho_{Z \subset X}=2 s_{1}^{4} s_{2}^{2} t_{1}^{3} t_{2}$. 
Definition 12 Let $(X, B)$ be a finite birack with birack rank $N, L$ a link of $c$ components and $W=\left(\mathbb{Z}_{N}\right)^{c}$. The birack polynomial enhanced invariant of $L$ with respect to $(X, B)$ is the multiset

$$
\phi_{(X, B)}^{\rho, M}(L)=\left\{\rho_{\operatorname{Im}(f) \subset X} \mid f \in \operatorname{Hom}(B R(L, \mathbf{w}),(X, B)), \mathbf{w} \in W\right\} ;
$$

in polynomial form we have

$$
\phi_{(X, B)}^{\rho}(L)=\sum_{\mathbf{w} \in W}\left(\sum_{f \in \operatorname{Hom}(B R(L, \mathbf{w}),(X, B))} z^{\rho_{\operatorname{Im}(f) \subset X}}\right) .
$$

Example 11 The polynomial enhanced invariant can distinguish labelings with image subbiracks which happen to have the same cardinality but are not isomorphic or are isomorphic but embedded differently in the overall birack. The birack in example 10 has birack rank 2. The unknot has six labelings by this birack over a period of writhes $w \in \mathbb{Z}_{2}$ as pictured, with four labelings occurring in writhe $w=0$ and two occurring in writhe $w=1$.
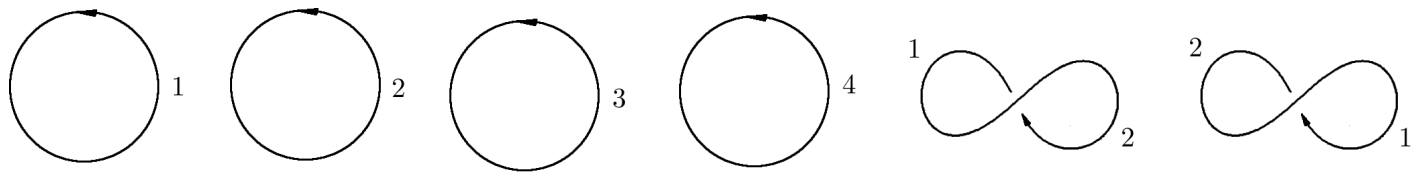

We have

$$
\phi_{(X, B)}^{\rho}(\text { Unknot })=4 z^{s_{1}^{2} t_{1}^{2} t_{2}^{2}+s_{2}^{2} t_{1}^{2} t_{2}^{2}}+2 z^{2 s_{1}^{4} s_{2}^{2} t_{1}^{3} t_{2}} .
$$

Remark 10 It is easy to see that the integral counting invariant $\phi_{(X, B)}^{\mathbb{Z}}$ can be recovered from $\phi_{(X, B)}^{\rho}(L)$ by specializing $z=1$ and that the image-enhanced invariant can be recovered by setting $s_{i}=t_{i}=1$. When $(X, B)$ is a quandle, $\phi_{(X, B)}^{\rho}(L)$ satisfies

$$
\phi_{(X, B)}^{\rho}(L)=\Phi_{q p}(L)\left(s_{2} t_{2}\right)^{|X|}
$$

where $\Phi_{q p}(L)$ is the quandle polynomial invariant with $s=s_{1}$ and $t=t_{1}$.

Example 12 To see that the polynomial-enhanced invariant is stronger than the counting invariant, we compute (using python code) that the knots $5_{1}$ and $6_{1}$ both have counting invariant 30 with respect to the 10-element birack with matrix listed below, but are distinguished by the image-enhanced invariant values

$$
\phi_{(X, B)}^{\rho}\left(5_{1}\right)=4 z^{s_{1}^{6} s_{2}^{10} t_{1} t_{2}^{5}}+5 z^{s_{1}^{2} s_{2}^{6} t_{1}^{6} t_{2}^{10}}+z^{s_{1}^{6} s_{2}^{10} t_{1}^{6} t_{2}^{10}}+20 z^{5 s_{1}^{2} s_{2}^{6} t_{1}^{6} t_{2}^{10}}
$$

and

$M_{B}=\left[\begin{array}{cccccccccc|ccccccccccc}1 & 1(X, B) \\ 1 & 3 & 5 & 2 & 4 & 1 & 1 & 1 & 1 & 1 & 1 & 1 & 1 & 1 & 1 & 1 & 1 & 1 & 1 & 1 \\ 5 & 2 & 4 & 1 & 3 & 2 & 2 & 2 & 2 & 2 & 2 & 2 & 2 & 2 & 2 & 2 & 2 & 2 & 2 & 2 \\ 4 & 1 & 3 & 5 & 2 & 3 & 3 & 3 & 3 & 3 & 3 & 3 & 3 & 3 & 3 & 3 & 3 & 3 & 3 & 3 \\ 3 & 5 & 2 & 4 & 1 & 4 & 4 & 4 & 4 & 4 & 4 & 4 & 4 & 4 & 4 & 4 & 4 & 4 & 4 & 4 \\ 2 & 4 & 1 & 3 & 5 & 5 & 5 & 5 & 5 & 5 & 5 & 5 & 5 & 5 & 5 & 5 & 5 & 5 & 5 & 5 \\ 7 & 7 & 7 & 7 & 7 & 6 & 10 & 9 & 8 & 7 & 8 & 8 & 8 & 8 & 8 & 6 & 6 & 6 & 6 & 6 \\ 9 & 9 & 9 & 9 & 9 & 8 & 7 & 6 & 10 & 9 & 6 & 6 & 6 & 6 & 6 & 7 & 7 & 7 & 7 & 7 \\ 6 & 6 & 6 & 6 & 6 & 10 & 9 & 8 & 7 & 6 & 9 & 9 & 9 & 9 & 9 & 8 & 8 & 8 & 8 & 8 \\ 8 & 8 & 8 & 8 & 8 & 7 & 6 & 10 & 9 & 8 & 7 & 7 & 7 & 7 & 7 & 9 & 9 & 9 & 9 & 9 \\ 10 & 10 & 10 & 10 & 10 & 9 & 8 & 7 & 6 & 10 & 10 & 10 & 10 & 10 & 10 & 10 & 10 & 10 & 10 & 10\end{array}\right]$


Remark 11 All of the invariants described in this paper are defined for virtual knots and links as well as classical knots and links via the usual method of ignoring virtual crossings.

Definition 13 For each of the invariants described above, the unlink with $c$ components $U_{c}$ can have values which look nontrivial. Thus, to aid in identifying nontrivial values of the invariants, for each invariant $\phi_{(X, B)}^{*}(L)$ we define the corresponding normalized birack invariant $\bar{\phi}_{(X, B)}^{*}(L)$ to be the difference between the invariant value on $L$ and the invariant value on the unlink of the corresponding number of components, i.e.

$$
\bar{\phi}_{(X, B)}^{*}(L)=\phi_{(X, B)}^{*}(L)-\phi_{(X, B)}^{*}\left(U_{c}\right) .
$$

Remark 12 The author's python code for computing these invariants is available at www .esotericka.org. The code represents biracks as pairs of square matrices and knot or link diagrams as Kauffman-style signed Gauss codes. The algorithm for finding all birack homomorphisms uses a working list of partly filled-in image vectors, selecting a blank entry and trying out all possible values while filling in other entries using the homomorphism conditions. Any such working image vectors with contradictory entries are discarded; any remaining image vectors with blanks are appended to the working list, and those with no remaining blanks are moved to an output list.

\section{Questions}

In this section we collect questions for future research.

It is clear that we can combine various enhancement strategies to define potentially stronger, if more unwieldy, multivariable enhanced invariants. Computer experiments using python cod® $\rrbracket^{1}$ suggest that the writhe enhancement is the most effective of the three enhancements considered in this paper for knots and links with small crossing number using small-cardinality biracks. Does this remain true when the crossing number or size of the target birack is increased?

$(t, s, r)$-biracks generalize Alexander biquandles and $(t, s)$-racks. What birack definition generalizes bilinear biquandles and symplectic quandles? What is the generalization of Coxeter racks to the birack case? What about other new types of biracks?

In both [14 and [17] it is shown that every quandle has a presentation as a quotient of a multiplicative Alexander quandle structure on the automorphism group of the quandle. Is there an analogous construction for biracks? The straightforward generalization does not work, but perhaps there is a non-obvious solution.

Future work will undoubtedly include generalizing the Yang-Baxter cocycle invariants and quandle cocycle invariants studied in papers like [5] and [6] to the birack case. More generally, though, we would like to see additional enhancements of the various counting invariants defined. We would also like to see improved algorithms for computing these invariants quickly. Functorial (as opposed to representational) invariants of biracks would be of interest as well.

\section{References}

[1] N. Andruskiewitsch and M. Graña. From racks to pointed Hopf algebras. Adv. Math. 178 (2003) 177-243.

[2] A. Bartholomew and R. Fenn. Biquandles of Small Size and some Invariants of Virtual and Welded Knots. arXiv:1001.5127

[3] S. Budden and R. Fenn. The equation $[B,(A-1)(A, B)]=0$ and virtual knots and links. Fund. Math. 184 (2004) 19-29.

[4] S. Budden and R. Fenn. Quaternion algebras and invariants of virtual knots and links. II. The hyperbolic case. J. Knot Theory Ramifications 17 (2008) 305-314.

\footnotetext{
1 available at www.esotericka.org
} 
[5] J. S. Carter, D. Jelsovsky, S. Kamada, L. Langford and M. Saito. Quandle cohomology and state-sum invariants of knotted curves and surfaces. Trans. Am. Math. Soc. 355 (2003) 3947-3989.

[6] J. Ceniceros and S. Nelson. Virtual Yang-Baxter 2-cocycle invariants. Trans. Amer. Math. Soc. 361 (2009) 5263-5283.

[7] J. Ceniceros and S. Nelson. $(t, s)$-racks and their link invariants arXiv:1011.5455

[8] J.C. Conway and G.C. Wraith, correspondence, 1959.

[9] R. Fenn, M. Jordan-Santana and L. Kauffman. Biquandles and virtual links. Topology Appl. 145 (2004) $157-175$.

[10] R. Fenn and C. Rourke. Racks and links in codimension two. J. Knot Theory Ramifications 1 (1992) 343-406.

[11] R. Fenn, C. Rourke and B. Sanderson. Trunks and classifying spaces. Appl. Categ. Structures 3 (1995) 321356 .

[12] R. Fenn and V. Turaev. Weyl Algebras and Knots. J. Geom. Phys. 57 (2007) 13131324.

[13] A. Henrich and S. Nelson, Semiquandles and flat virtual knots. arXiv:0901.4315.

[14] D. Joyce. A classifying invariant of knots, the knot quandle. J. Pure Appl. Algebra 23 (1982) 37-65.

[15] L. H. Kauffman and D. Radford. Bi-oriented quantum algebras, and a generalized Alexander polynomial for virtual links. Contemp. Math. 318 (2003) 113-140.

[16] D. Lam and S. Nelson. An isomorphism theorem for Alexander biquandles. Internat. J. Math. 20 (2009) $97-107$.

[17] S. V. Matveev. Distributive groupoids in knot theory. Math. USSR, Sb. 47 (1984) 73-83.

[18] S. Nelson, Classification of finite Alexander quandles. Topol. Proc. 27 (2003) 245-258.

[19] E. A. Navas and S. Nelson. On symplectic quandles. Osaka J. Math 45 (2008) 973-985.

[20] S. Nelson, A polynomial invariant of finite quandles. J. Alg. Appl. 7 (2008) 263-273.

[21] S. Nelson. Generalized quandle polynomials. arXiv:0801.2979 to appear in Can. Bull. Math.

[22] S. Nelson and R. Weighard, Link invariants from finite Coxeter racks, arXiv:0808.1584

[23] S. Nelson. Link invariants from finite racks. arXiv:0808.0029

[24] S. Nelson and J. Vo. Matrices and Finite Biquandles. Homology, Homotopy Appl. 8 (2006) 51-73.

[25] M. Takasaki. Abstractions of symmetric functions. Tohoku Math. J. 49 (1943) 143-207.

Department of Mathematical Sciences

Claremont McKenna College

850 Columbia Ave.

Claremont, CA 91711

Email: knots@esotericka.org 